\title{
Investigations of the static and dynamic characteristics of the precision hydrostatic spindle with mid- thrust bearing under different loads Chenggang Fang ${ }^{1 *}$, Wucheng You ${ }^{2}$, Dehong Huo
}

Keywords: Precision hydrostatic spindle, five degree-of-freedom, coupled journal and thrust bearings, static and dynamic coefficients, stability

\section{Abstract:}

The purpose of this paper is to investigate the static and dynamic characteristics of the precision hydrostatic spindle with midthrust bearing under different working conditions. Firstly, the paper establishes the fluid governing equations of the coupled journal and thrust bearings based on orifice restrictors; and it solves the dynamic and static Reynolds equations with the perturbation method and the finite difference method to obtain the steady and transient pressure distribution functions. Then the stiffness and damping characteristics matrixes of the spindle are obtained by integrating steady and transient pressure. Furthermore, by establishing the motion equation of the spindle rotor with five degree-of-freedom, the quasi-static equilibrium position and stability criterion of the rotor under different working conditions are solved. Finally, the relationship between the dynamic and static characteristics of the spindle and the cutting force, rotational speed, and cutting distance is simulated and analyzed. The simulation results show the variant rules of the performance indexes such as stiffness, damping, quasi-static position, and stability of the spindle under different working conditions, which provides an important design consideration for the precision hydrostatic spindle.

\section{Introduction}

Precision hydrostatic spindles have the following advantages: enormous stiffness and capacity, high rotational accuracy, superior vibration damping, fewer power losses, lower requirements regarding the machining accuracy, long life span, etc. [1]. It can be widely used in various precision machining occasions, such as precision turning, milling, grinding. Hydrostatic spindles usually adopt a structure with frontthrust bearing, which has the advantages of easy to manufacture and assemble. However, the radial stiffness of this type of spindle is lower than other varieties. Some researchers proposed to improve the spindle stiffness by increasing the diameter of the thrust bearing, which also increases the radial dimension of the spindle [2]. The midthrust bearing structure discussed in some researches can effectively improve the stiffness of the spindle without increasing the structure size of the spindle $[3,4,5,6]$.

A mathematical analytical model of the flow field based on the Reynolds equation and the flow continuity equation was employed to accurately analyze the performance of the hydrostatic spindle with the coupled journal and thrust bearing [7]. Methods such as physical perturbation and mathematics perturbation are often adopted to obtain the stiffness and damping matrix of the hydrostatic spindle quickly and accurately [8,9]. The algorithms such as finite difference method (FDM), finite element method (FEM), and finite volume method (FVM) are usually employed to solve partial differential equations. In the above algorithms, FDM is often applied in the analysis and calculation of hydrostatic bearing due to its simple programming and high computational efficiency. Considering that hydrostatic bearing often adopts the multi-recess oil supply structure, the internal flow effect between different recess requires repeated flow balance iteration in the process of solving the pressure distribution function. A decoupling algorithm based on the Reynolds equation for multi-recess and laminar hydrostatic bearing is proposed by $\mathrm{Du}$ and Liang, which improves the computational efficiency effectively ${ }^{[10]}$.

Besides, the dynamic and static characteristics of the machine tool spindle directly determine the accuracy and stability of the cutting process; and that is a crucial design performance index. For the hydrostatic spindle, the dynamic and static characteristics will vary significantly with the external load conditions due to the rotor attitude change of the spindle. $\mathrm{Li}$ and Liu studied spindle rotation accuracy under different cutting loads, imbalance response, and stability, and pointed out that the tilt of the spindle rotor has a distinct impact on the above indexes [12]. Shi et. al researched the characteristics of the five degree-of-freedom aerostatic spindles of fly-cutting machine tools, and the conclusion showed the pressure distribution, stiffness, and damping coefficients of the spindle had significant variation due to the changes of the spindle rotation speed and tilt angle ${ }^{[13]}$. Abdurrahim studied the variation rules of air film thickness, pressure distribution, and bearing capacity of the spindle under the combined action of different inclination angles, rotating speed, and eccentricity [14]. Myunggyu focused on the influence of the spindle rotor angular displacement on the spindle's dynamic performance, obtained the relationship between stiffness, damping coefficients, and the rotor angular displacement, and pointed out that the tilting motion plays an integral role in the determination of both the force and the moment coefficients [15,16].

However, the existing literature usually studies the static and dynamic characteristics of the hydrostatic spindle with the front-thrust bearing by supposing the spindle rotor is under a pretilt angle, and very few performed comprehensive studies on dynamic and static features of the hydrostatic spindle with the mid-thrust bearing exerted by the actual 3D cutting load. This paper establishes a mathematical model of the hydrostatic spindle with mid-thrust bearing and introduces the linear superposition solution of the flow continuity equation. Afterward, it solves the dynamic Reynolds equation by the perturbation method to obtain the performance parameters such as pressure distribution, bearing capacity, flowrate, stiffness, and damping of the spindle. Furthermore, by establishing the motion equation of the rotor with five degree-of-freedom, the quasi-static equilibrium position and the critical stability of the rotor are solved by the Euler method. Finally, the paper studies the dynamic and static characteristics of the spindle under different cutting force, rotation speed, and load distance by simulation, which provides a theoretical basis for the design and experiment of the hydrostatic spindle.

1 School of Mechanical and Power Engineering, Nanjing Tech University, No.30, Puzhu Southern Road, Pukou District, Nanjing 211816, Jiangsu, China

2 Nanjing High Accurate Marine Equipment Co., Ltd, No.1 Baoding Road, Science garden, Jiangning, Nanjing 211816, Jiangsu, China

3 Mechanical Engineering, School of Engineering, Newcastle University Newcastle, UK, NE1 7RU

*Corresponding author: Chenggang Fang,

Email: fangchenggang53@gmail.com 


\section{Theoretical model}

\subsection{Governing Equations}

At first, the following assumptions are made to establish an analytical model for the externally pressurized journal and thrust bearings of the hydrostatic spindle:

(1) The oil lubricant is an isothermal Newtonian fluid due to the low-temperature rise and the inflow of oil at a constant temperature.

(2) The liquid is in laminar flow.

(3) The oil is incompressible and satisfies the flow continuity equation.

(4) Gravity and inertial forces are negligible.

(5) Variation of pressure at the direction of the oil film thickness is negligible.

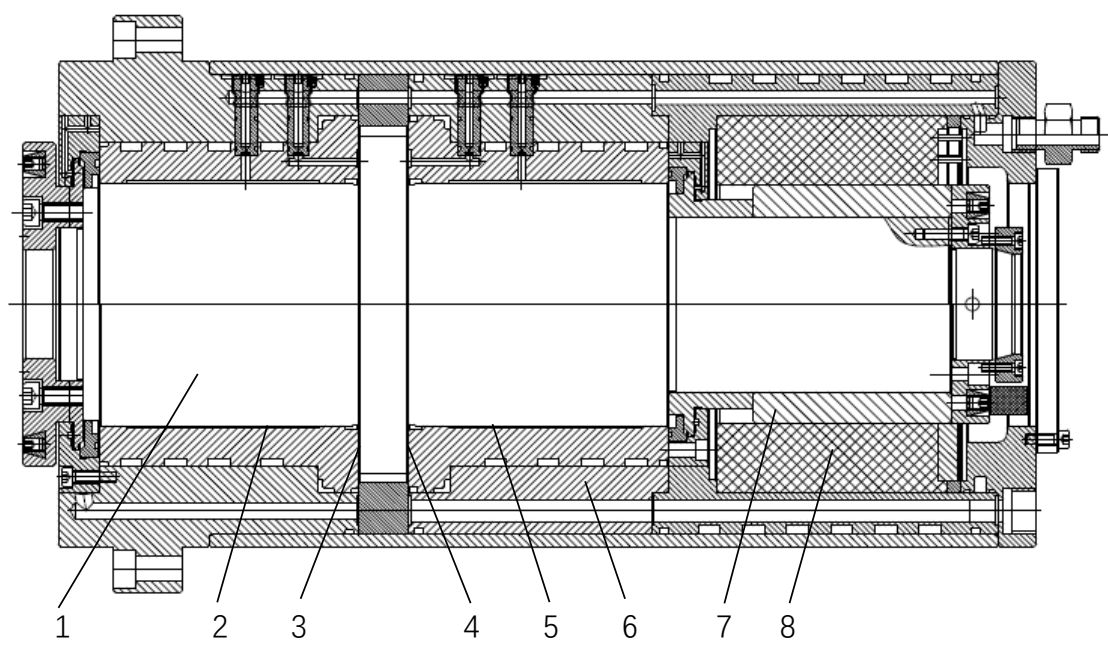

Figure 1. The hydrostatic motorized spindle. (1) rotor of the spindle; (2) front journal bearing; (3) front thrust bearing; (4) rear thrust bearing; (5) rear journal bearing; (6) shell; (7) rotor of the motor; (8) stator of the motor.
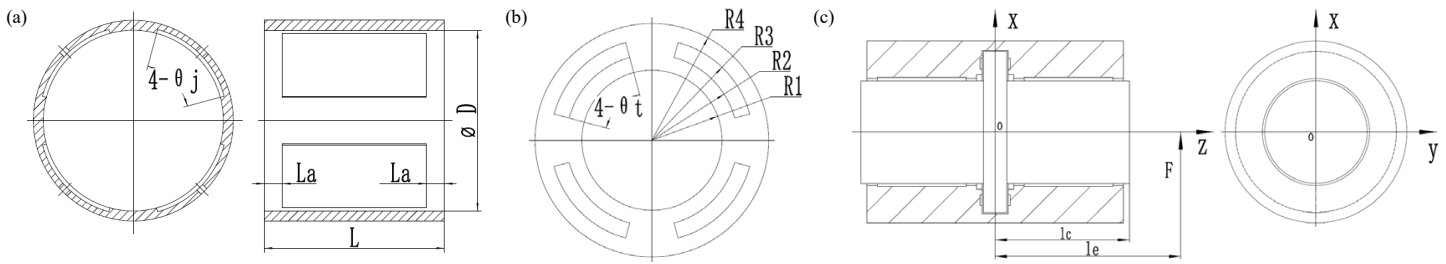

Figure 2. Schematic view of the journal bearing and thrust bearing. (1) The geometry of the journal bearing; (2) geometry of the thrust bearing; (3) cartesian coordinate system of the spindle.

Figure 1 shows a schematic view of a precision hydrostatic spindle with a mid-thrust bearing. The spindle mainly includes a pair of journal bearings (2) and (5), and a pair of thrust bearings (3) and (4), which are supported on the spindle shell (6). The torque motor with the rotor (7) and stator (8) drives the spindle rotor to provide cutting power. Figures 2(a) and 2(b) show the schematic view of the oil-lubricated journal bearing and thrust bearing respectively. The externally pressurized oil flows through the orifice restrictors into the bearing clearance and then flows out into the ambient air. Generalized Reynolds equations in the cartesian coordinate system, governing the fluid flow field in the clearance space of the journal and thrust bearings are [17]:

$\frac{\partial}{\partial x}\left(h^{3} \frac{\partial p}{\partial x}\right)+\frac{\partial}{\partial z}\left(h^{3} \frac{\partial p}{\partial z}\right)=6 \eta U \frac{\partial h}{\partial x}+12 \eta \frac{\partial h}{\partial t}$

$\frac{\partial}{\partial r}\left(r h^{3} \frac{\partial p}{\partial r}\right)+\frac{\partial}{\partial \theta}\left(\frac{h^{3}}{r} \frac{\partial p}{\partial \theta}\right)=6 \eta r \omega \frac{\partial h}{\partial \theta}+12 \eta r \frac{\partial h}{\partial t}$

where $p$ is the pressure distribution; $h$ is the oil film thickness; $x$ and $z$ are the coordinate axes; $\theta$ is angular coordinate; $\eta$ is the viscosity of oil; $U$ is the circumferential velocity of the journal bearing; $\omega$ is the spindle speed; $r$ is the radius of thrust bearing; $t$ is time.

The following dimensionless parameters are introduced: $\theta=\frac{x}{R}, \bar{z}=\frac{z}{R}, \quad \bar{p}=\frac{p}{p_{s}}, \bar{h}=\frac{h}{c_{0}}, \bar{t}=\omega t, U=R \omega, S_{h}=$

$\frac{6 \eta \omega}{p_{s}}\left(\frac{R}{C_{0}}\right)^{2}, \quad \bar{R}=\frac{r}{R_{4}}, \quad S_{t}=\frac{6 \eta \omega}{p_{s}}\left(\frac{R_{4}}{C_{0}}\right)^{2}$

where $R$ is the radius of journal bearing; $p_{s}$ is oil supply pressure; $R_{4}$ is the outer radius of the thrust bearing; $C_{0}$ is the designed oil film thickness

Then the equations (1) and (2) in dimensionless form can be written as:

$$
\begin{aligned}
& \frac{\partial}{\partial \theta}\left(\bar{h}^{3} \frac{\partial \bar{p}}{\partial \theta}\right)+\frac{\partial}{\partial \bar{z}}\left(\bar{h}^{3} \frac{\partial \bar{p}}{\partial \bar{z}}\right)=S_{h} \frac{\partial \bar{h}}{\partial \theta}+2 S_{h} \frac{\partial \bar{h}}{\partial \bar{t}} \\
& \frac{\partial}{\partial \bar{R}}\left(\bar{R} \bar{h}^{3} \frac{\partial \bar{p}}{\partial \bar{R}}\right)+\frac{\partial}{\partial \theta}\left(\frac{\bar{h}^{3}}{\bar{R}} \frac{\partial \bar{p}}{\partial \theta}\right)=S_{t} \bar{R} \frac{\partial \bar{h}}{\partial \theta}+2 S_{t} \bar{R} \frac{\partial \bar{h}}{\partial \bar{t}}
\end{aligned}
$$

\subsection{0il film thickness}

In Figure 2(c), setting a coordinate system $(O-x y z)$ on the geometric symmetry center of the spindle shell, the rotor position in this coordinate system can be described as $\left(\Delta x, \Delta y, \Delta z, \Delta \theta_{x}, \Delta \theta_{y}\right)$. The oil film thickness of journal bearings at $z_{i}$ are:

$h=C_{0}-\left(\Delta x \pm z_{i} \Delta \theta_{y}\right) \cos \theta-\left(\Delta y \mp z_{i} \Delta \theta_{x}\right) \sin \theta$

where ' \pm ' in the second item and ' $\mp$ ' in the third item represent right and left journal bearing, respectively; $z_{i}$ is the axial coordinate of the journal bearing. The positive and negative directions of $\Delta \theta_{x}$ and $\Delta \theta_{y}$ refer to the right-hand screw rule.

Similarly, the oil film thickness of thrust bearings at $r_{i}$ can be written as:

$h=C_{0} \mp\left(\Delta z+\Delta \theta_{x} r_{i} \sin \theta+\Delta \theta_{y} r_{i} \cos \theta\right)$

where ' $\mp$ ' represents right and left bearing, respectively; $r_{i}$ is the radius of the thrust bearing.

Introducing dimensionless factors: 
$\Delta \bar{x}=\frac{\Delta x}{C_{0}}, \quad \Delta \bar{y}=\frac{\Delta y}{C_{0}}, \quad \Delta \bar{z}=\frac{\Delta z}{C_{0}}, \bar{Z}_{i}=\frac{z_{i}}{C_{0}}, \quad \bar{r}_{i}=\frac{r_{i}}{C_{0}}$

Substituting the above factors into equations (5) and (6), the dimensionless oil film thickness of journal and thrust bearings can be expressed as:

$\bar{h}=1-\left(\Delta \bar{x} \pm \bar{Z}_{i} \Delta \theta_{y}\right) \cos \theta-\left(\Delta \bar{y} \mp \bar{Z}_{i} \Delta \theta_{x}\right) \sin \theta$

$\bar{h}=1 \mp\left(\Delta \bar{z}+\Delta \theta_{x} \bar{r}_{i} \sin \theta+\Delta \theta_{y} \bar{r}_{i} \cos \theta\right)$

\subsection{Perturbation equations and boundary conditions}

When the spindle rotor oscillating near the quasi-static position, expanding the oil film thickness and pressure at the quasi-static position as Taylor expansion, retain the low-order term in dimensionless form as [18]:

$\bar{h}=\bar{h}_{0}+\sum \frac{\partial \bar{h}}{\partial \xi} \Delta \bar{\xi}+\sum \frac{\partial \bar{h}}{\partial \dot{\xi}} \Delta \bar{\xi}$

$\bar{p}(\theta, z)=\bar{p}_{0}+\sum \frac{\partial \bar{p}}{\partial \xi} \Delta \bar{\xi}+\sum \frac{\partial \bar{p}}{\partial \dot{\xi}} \Delta \overline{\dot{\xi}}$

where $\xi=x, y, z, \theta_{x}, \theta_{y}$.

By substituting equations (9) and (10) into equations (3) and (4), the Reynolds equations and its perturbation equations of the journal bearing is obtained as follows:

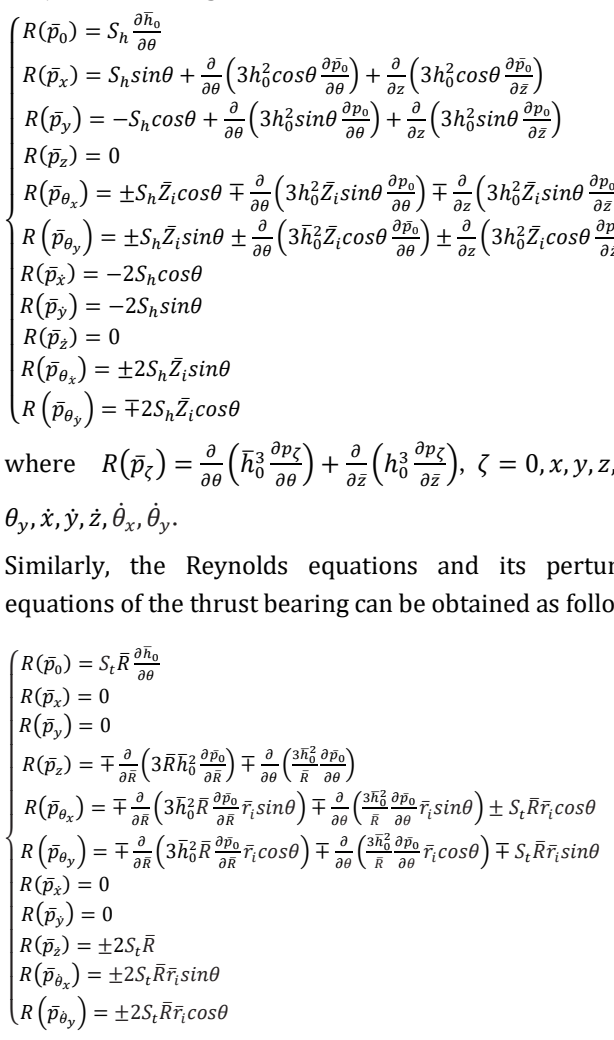

where $R\left(\bar{p}_{\zeta}\right)=\frac{\partial}{\partial \theta}\left(\frac{h_{0}^{3}}{\bar{R}} \frac{\partial p_{\zeta}}{\partial \theta}\right)+\frac{\partial}{\partial \bar{R}}\left(\bar{R} h_{0}^{3} \frac{\partial p_{\zeta}}{\partial \bar{R}}\right), \quad \zeta=0, x, y, z, \theta_{x}$, $\theta_{y}, \dot{x}, \dot{y}, \dot{z}, \dot{\theta}_{x}, \dot{\theta}_{y}$

Appropriate boundary conditions for the above equations are expressed as:

1) The outlet pressure is $P=0$;

2) The symmetric boundary condition is $\frac{d P}{d Z}=0$;

3) The periodic boundary condition is $P(0, z)=P(2 \pi, z)$;

4) The pressure in each recess is assumed uniform;

5) The outlet perturbed pressures are $\bar{p}_{\zeta}=0 \quad(\zeta=$ $\left.0, x, y, z, \theta_{x}, \theta_{y}, \dot{x}, \dot{y}, \dot{z}, \dot{\theta}_{x}, \dot{\theta}_{y}\right)$.

\subsection{Flow continuity equation}

To solve the Reynolds equations of the journal and thrust bearings compensated by orifice restrictors, the boundary condition of mass flowrate through orifices is considered.

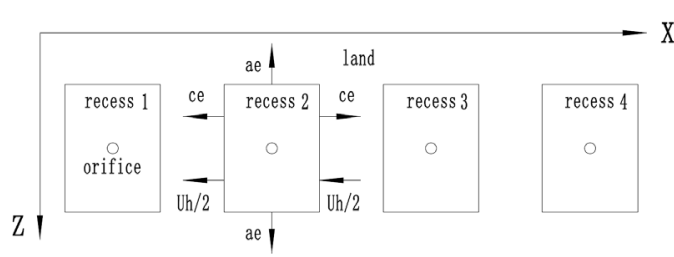

Figure 3. The unwrapped flow field of bearing As shown in Figure 3, there are four uniform recesses arranged in the circumference direction of the journal and thrust bearing, and the orifice restrictors are in the center of the recesses. The flowrate through orifice restrictor $Q_{\text {in }}$ can be written as [19]:

$Q_{\text {in }}=\alpha \frac{\pi d_{c}^{2}}{4} \sqrt{\frac{2 \Delta p}{\rho}}$

where $\alpha$ is flowrate coefficient $(\alpha=0.6-0.7) ; d_{c}$ is the diameter of the orifice restrictor; $\Delta p$ is the differential pressure flowing through the orifice restrictor; $\rho$ is the density of oil.

The flowrate through the film-land around the recess $Q_{\text {out }}$ can be described as:

$Q_{\text {out }}=\int_{a e} \frac{h^{3}}{12 \eta} \frac{d p}{d z} d x+\int_{c e}\left( \pm \frac{U h}{2}+\frac{h^{3}}{12 \eta} \frac{d p}{d x}\right) d z+A_{r} \frac{\partial h}{\partial t}$

where $A_{r}$ is the recessed area; ae represents the axial edges of recess; $c e$ represents the circumferential edges of recess. Introducing factor $\frac{p_{s} C_{0}^{3}}{12 \eta}$ into equations (13) and (14) of which can be rewritten in dimensionless form respectively as:

$\bar{Q}_{\text {in }}=\delta \sqrt{1-\bar{p}}$

$\bar{Q}_{\text {out }}=\int_{a e} \bar{h}^{3} \frac{\partial \bar{p}}{\partial \bar{z}} d \theta+\int_{c e}\left( \pm S_{h} \bar{h}+\bar{h}^{3} \frac{\partial \bar{p}}{\partial \theta}\right) d \bar{z}+2 S_{h} \frac{\partial \bar{h}}{\partial \bar{t}} d \theta d \bar{z}$

where $\delta$ is the dimensionless flowrate coefficient of the

orifice restrictor, $\delta=\frac{3 \sqrt{2} \pi \alpha d_{c}^{2} \eta}{\mathrm{C}_{0}^{3} \sqrt{\rho p_{s}}}$.

For incompressible laminar fluid, substituting the Taylor expansion of pressure, equation (10), into equation (16) and decomposing it, the zero and the first order of flow continuity equation can be obtained:

$\bar{Q}_{\text {in }}^{\zeta}=\bar{Q}_{\text {out }}^{\zeta}, \zeta=0, x, y, z, \theta_{x}, \theta_{y}, \dot{x}, \dot{y}, \dot{z}, \dot{\theta}_{x}, \dot{\theta}_{y}$

\subsection{Static and dynamic characteristics}

The static characteristics of the spindle include load capacity, load moments, and the dynamic characteristics are evaluated considering the stiffness and damping coefficients. The steady pressure $\bar{p}_{0}(\theta, \bar{z})$ and transient pressure $\bar{p}_{\zeta}(\theta, \bar{z}, t)$ caused by the displacement and velocity of the rotor can be solved by the coupled perturbation Reynolds equations and flow continuity equation. The algorithm of FDM is employed to solve equations (11) to (17) to obtain the results of the aforementioned characteristic parameters. Also, this study adopts a mathematical perturbation method to solve the above equations because its efficiency and accuracy are higher than that of the physical perturbation method. The process is as follows.

Firstly, the dimensionless load capacity and load moments $\left[\bar{F}_{x}, \bar{F}_{y}, \bar{F}_{z}, \bar{M}_{x}, \bar{M}_{y}\right]^{T}$ can be calculated by integrating steady pressure $p_{0}(\theta, \bar{z})$ as:

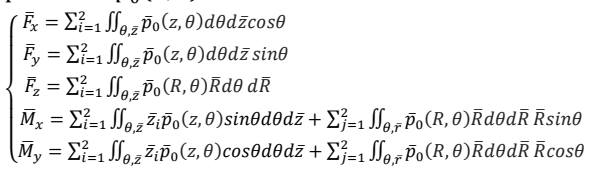

where $i$ and $j$ represent the number of journal and thrust bearings; $\quad \bar{p}_{0}(z, \theta)$ and $\bar{p}_{0}(R, \theta)$ are the pressure 
distribution respectively of the journal and thrust bearing. Secondly, the dimensionless dynamic characteristics coefficients of each bearing can be calculated by integrating transient pressure $\bar{p}_{\zeta}(\theta, \bar{z}, t)$ across the oil film of the coupled journal and thrust bearings. Further, the total stiffness $\bar{K}$ of the spindle is the sum of the stiffness of each bearing as follows ${ }^{[20,21]}$ :

$\bar{K}=\bar{K}_{j r}+\bar{K}_{j l}+\bar{K}_{t r}+\bar{K}_{t l}$

where $\bar{K}_{j r}, \bar{K}_{j l}, \bar{K}_{t r}, \bar{K}_{t l}$ represent the journal and thrust bearing stiffness matrixes on the left and right sides, respectively.

In the same way, the total damping $\bar{C}$ of the spindle is the sum of the damping of each bearing:

$\bar{C}=\bar{C}_{j r}+\bar{C}_{j l}+\bar{C}_{t r}+\bar{C}_{t l}$

where $\bar{C}_{j r}, \bar{C}_{j l}, \bar{C}_{t r}$, and $\bar{C}_{t l}$ are respectively the journal and thrust bearing damping matrixes on the left and right sides.

Since the calculation method of the journal bearing is the same as that of thrust bearings, take right journal bearings as an example to calculate its stiffness and damping matrix as follows:

$$
\begin{gathered}
\bar{K}_{j r}=\left[\begin{array}{llll}
\bar{k}_{x x} & \bar{k}_{x y} & \bar{k}_{x \theta_{x}} & \bar{k}_{x \theta_{y}} \\
\bar{k}_{y x} & \bar{k}_{y y} & \bar{k}_{y \theta_{x}} & \bar{k}_{y \theta_{y}} \\
\bar{k}_{\theta_{x} x} & \bar{k}_{\theta_{x} y} & \bar{k}_{j \theta_{x} \theta_{x}} & \bar{k}_{j \theta_{x} \theta_{y}} \\
\bar{k}_{\theta_{y^{x}}} & \bar{k}_{\theta_{y} y} & \bar{k}_{j \theta_{y} \theta_{x}} & \bar{k}_{j \theta_{y} \theta_{y}}
\end{array}\right]= \\
\iint_{\theta, \bar{z}}\left[\begin{array}{c}
\cos \theta \\
\sin \theta \\
\bar{z}_{\cdot} \cdot \sin \theta \\
\bar{z}_{i} \cdot \cos \theta
\end{array}\right]\left[\begin{array}{llll}
\bar{x}_{x r} & \bar{p}_{y r} & \bar{p}_{\theta_{x r}} & \bar{p}_{\theta_{y r}}
\end{array}\right] d \theta d \bar{z}
\end{gathered}
$$

$\bar{C}_{j r}=\left[\begin{array}{cccc}\bar{c}_{x x} & \bar{c}_{x y} & \bar{c}_{x \theta_{x}} & \bar{c}_{x \theta_{y}} \\ \bar{c}_{y x} & \bar{c}_{y y} & \bar{c}_{y \theta_{x}} & \bar{c}_{y \theta_{y}} \\ \bar{c}_{\theta_{x} x} & \bar{c}_{\theta_{x} y} & \bar{c}_{j \theta_{x} \theta_{x}} & \bar{c}_{\theta_{x} \theta_{y}} \\ \bar{c}_{\theta_{y} x} & \bar{c}_{\theta_{y} y} & \bar{c}_{j \theta_{y} \theta_{x}} & \bar{c}_{j \theta_{y} \theta_{y}}\end{array}\right]=$

$$
\iint_{\theta, \bar{z}}\left[\begin{array}{c}
\cos \theta \\
\sin \theta \\
\bar{z}_{z^{\prime}} \cdot \sin \theta \\
\bar{z}_{i} \cdot \cos \theta
\end{array}\right]\left[\begin{array}{llll}
\bar{p}_{x r} & \bar{p}_{y r} & \bar{p}_{\dot{\theta}_{x} r} & \bar{p}_{\dot{\theta}_{y} r}
\end{array}\right] d \theta d \bar{z}
$$

where $\left[\begin{array}{llll}\bar{p}_{x r} & \bar{p}_{y r} & \bar{p}_{\theta_{x r}} & \bar{p}_{\theta y r}\end{array}\right]$ are the transient pressure caused by displacement; $\left[\begin{array}{llll}\bar{x}_{x r} & \bar{p}_{y r} & \bar{p}_{\dot{\theta}_{x} r} & \bar{p}_{\dot{\theta}_{y} r}\end{array}\right]$ are the transient pressure caused by velocity.

Furthermore, $\bar{K}_{j l}, \bar{K}_{t r}, \bar{K}_{t l}$ and $\bar{C}_{j l}, \bar{C}_{t r}, \bar{C}_{t l}$ in equations (19) and (20) can be solved in the same method as that in equations (21) and (22); the process will not be repeated here.

\subsection{Static equilibrium position}

In the quasi-equilibrium state, the static equilibrium equation of the spindle rotor must satisfy the following equilibrium condition [22]:

$\left[\begin{array}{c}F_{x} \\ F_{y} \\ F_{z} \\ M_{x} \\ M_{y}\end{array}\right]=\left[\begin{array}{c}F_{x e} \\ F_{y e}+m g \\ F_{z e} \\ F_{y e} \cdot l_{e} \\ F_{x e} \cdot l_{e}+F_{z e} \cdot R\end{array}\right]$

where $\left[\begin{array}{lllll}F_{x} & F_{y} & F_{z} & M_{x} & M_{y}\end{array}\right]^{T}$ are the oil film forces and moments on the spindle rotor, which can be solved from equation (18); $F_{x e}, F_{y e}, F_{z e}$ are components of cutting force along with the $x / y / z$ coordinates. For the sake of simplicity, taking a turning tool with a principal cutting edge angle of $45^{\circ}$ as an example, the component of cutting forces satisfy the following relation: $F_{x e}=0.45 F_{y e}, F_{z e}=0.35 F_{y e}{ }^{[23]}, l_{e}$ is the distance between the main cutting force and the center of the spindle rotor.

When the spindle is in the steady-state equilibrium position, the oil film forces and moments on the spindle rotor are equal to the equivalent spring force of the oil film, i.e.

$F(X)=K \Delta X$

where $\Delta X=\left[\begin{array}{lllll}\Delta x & \Delta y & \Delta z & \Delta \theta_{x} & \Delta \theta_{y}\end{array}\right]^{T}$ is the displacement of the spindle rotor from a steady-state equilibrium position; $K$ is the total stiffness matrix of the spindle.

Set the right item of equation (23) as $F_{w}$, then equation (23) can be rewritten in iterative form as:

$f(X)=F(X)-F_{w}=0$

To solve equation (25), create an iterative function based on the Newton-Raphson algorithm as:

$X_{i+1}=X_{i}+\epsilon K^{-1} f\left(X_{i}\right)$

where $\epsilon$ is the relaxation factor.

The convergence criteria of equations (24) and (25) are employed as $\sum\left|X_{i+1}-X_{i}\right|<10^{-6}$.

\subsection{Stability analysis}

The dynamics equations of the rigid rotor with five degree-offreedom supported by the coupled journal and thrust bearings, including both the translation and tilting motion, can be derived as follows ${ }^{[24,25]}$ :

$M \ddot{X}+(C+G) \dot{X}+K X=0$

$M=\left[\begin{array}{ccccc}\mathrm{m} & 0 & 0 & 0 & 0 \\ 0 & \mathrm{~m} & 0 & 0 & 0 \\ 0 & 0 & \mathrm{~m} & 0 & 0 \\ 0 & 0 & 0 & \mathrm{I}_{\mathrm{x}} & 0 \\ 0 & 0 & 0 & 0 & \mathrm{I}_{\mathrm{y}}\end{array}\right], G=\left[\begin{array}{ccccc}0 & 0 & 0 & 0 & 0 \\ 0 & 0 & 0 & 0 & 0 \\ 0 & 0 & 0 & 0 & 0 \\ 0 & 0 & 0 & 0 & \omega \mathrm{I}_{\mathrm{z}} \\ 0 & 0 & 0 & -\omega \mathrm{I}_{\mathrm{z}} & 0\end{array}\right]$ (27)

where $m$ is the mass of the rotor; $I_{x}, I_{y}$ and $I_{z}$ are the mass moments of inertia of the spindle rotor, respectively.

Set the homogeneous solution of equation (27) an exponential function $X=\left[\begin{array}{lllll}x_{h} & y_{h} & z_{h} & \theta_{x h} & \theta_{y h}\end{array}\right]^{T} e^{\lambda t}$, and substitute $\mathrm{X}$ into equation (27), and the equation can be simplified as follows:

$\lambda^{2} M+\lambda(C+G)+K=0$

The eigenvalue of equation (28) can be generally expressed as $\lambda=\sigma+j \omega_{s}$. The motion of the rotor is stable if $\sigma$ is smaller than zero, and it is unstable if $\sigma$ is higher than zero. So, the critical stable condition of rotor motion can be inferred as $\sigma=$ 0 . By substituting the eigenvalue $\lambda$ into equation (28), rewrite the equation as:

$K-M \omega_{s}^{2}+i \omega_{s}(C+G)=0$

Set equivalent stiffness $K_{e q}=M \omega_{s}^{2}$, whirl ratio $\gamma=\omega_{s} / \omega$. Introducing factor $p_{s} D$, the dimensionless form of $K_{e q}$ can be written as:

$\bar{K}_{e q}=\frac{M \omega_{s}^{2}}{p_{s} D}$

Set $\bar{\omega}^{2}=\frac{M \omega^{2}}{p_{s} D}$, and substitute it to equation (30), the dimensionless rotary speed of the spindle is:

$\bar{\omega}=\frac{\sqrt{\bar{K}_{e q}}}{\gamma}$

Applying the critical condition of the spindle rotor into equation (29), rearrange the real part and imaginary part of the corresponding determinant. Furthermore, equivalent stiffness $\bar{K}_{e q}$, whirl ratio $\gamma$, and dimensionless rotary speed $\bar{\omega}$ can be calculated from the equations of the real part and the imaginary part.

\section{Results and conclusions}

Based on the above theory, a computer program is developed by using MATLAB to carry out an analysis of the static and 
dynamic performances of the motorized spindle bearing system. The parameters of the oil-lubricated bearing and spindle are summarized in Tables 1,2 , and 3, respectively. The oil film was discretized by a rectangular grid with dimensionless intervals with about 135 grids in the circumferential direction and about 45 grids in the axial direction. A few validation tests were made with coarser grids of 4800 and finer grids of 7500 with different intervals, and the predicted results of the static characteristics differ by smaller than $0.2 \%$ from those obtained by the initial grid.

\begin{tabular}{lc}
\multicolumn{1}{c}{ Table 1. The specifications of the spindle ${ }^{[26]}$} \\
\hline \multicolumn{1}{c}{ Parameter name } & Value \\
\hline Rated speed $(\mathrm{rpm})$ & 3000 \\
Max speed $(\mathrm{rpm})$ & 4800 \\
Rated cutting force $(N)$ & 2000 \\
Max cutting force $(N)$ & 4000 \\
Rated power of the motor $(K W)$ & 3 \\
Mass of rotor $(\mathrm{m}, \mathrm{Kg})$ & 15 \\
Concentric pressure ratio $\left(\beta=\frac{p_{r}}{p_{s}}\right)$ & 0.65 \\
& 2.5 \\
Power ratio at max speed $\left(K_{p}\right)$ & 4 \\
Oil supply pressure $\left(p_{s}, M P a\right)$ & 0.002 \\
Oil viscosity $(\eta, P a \cdot s)$ & 20 \\
Oil supply temperature $\left({ }^{\circ} \mathrm{C}\right)$ &
\end{tabular}

Table 2. Parameters of the journal bearing [26]

\begin{tabular}{lc}
\hline \multicolumn{1}{c}{ Parameter name } & Value \\
\hline Orifice restrictor diameter $\left(d_{c}, \mathrm{~mm}\right)$ & 0.5 \\
Oil film thickness $\left(h_{0}, \mathrm{~mm}\right)$ & 0.016 \\
Diameter of journal $(D, \mathrm{~mm})$ & 90 \\
Bearing axial length $(L, \mathrm{~mm})$ & 90 \\
\hline
\end{tabular}

(a)

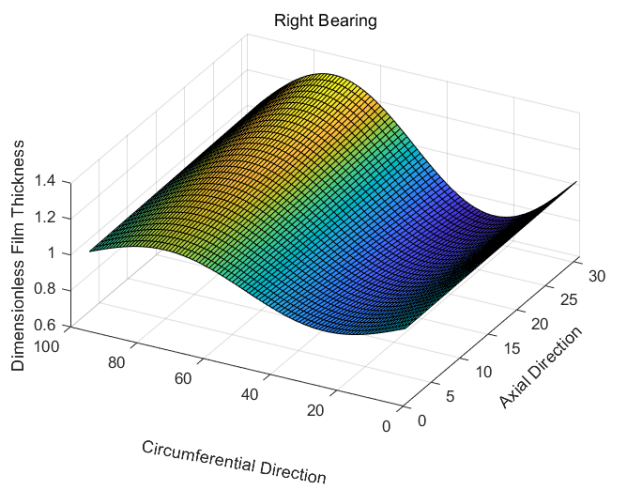

(c)

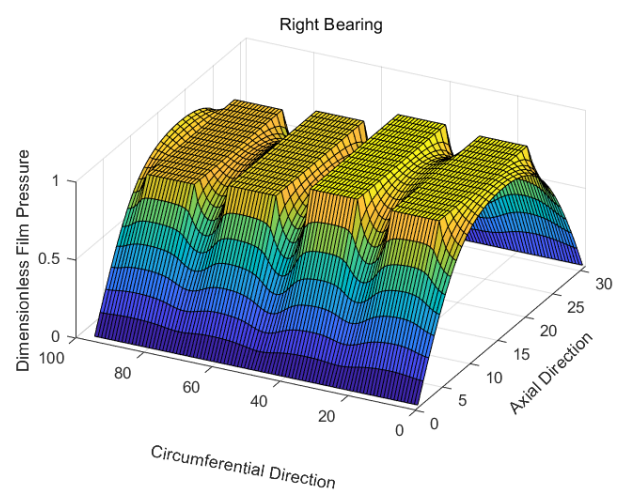

Length/diameter ratio $(L / D)$

Width of film land $\left(L_{a}, \mathrm{~mm}\right)$

Land-width ratio $\left(L_{a} / L\right)$

Inter-recess angle $\left(\theta_{j},{ }^{\circ}\right)$

Inter-recess land-width ratio

0.33

Table 3. Parameters of the thrust bearing

\begin{tabular}{lc}
\hline \multicolumn{1}{c}{ Parameter name } & Value \\
\hline Orifice restrictor diameter $(\mathrm{mm})$ & 0.7 \\
Oil film thickness $\left(h_{0}, \mathrm{~mm}\right)$ & 0.016 \\
Inner radius of thrust bearing $(R 1, \mathrm{~mm})$ & 48 \\
Inner radius of recess $(R 2, \mathrm{~mm})$ & 52 \\
Outer radius of recess $(R 3, \mathrm{~mm})$ & 58 \\
Outer radius of thrust bearing $(R 4, \mathrm{~mm})$ & 62 \\
Land-width ratio $((R 2-R 1) /(R 4-R 1))$ & 0.28 \\
Inter-recess angle $\left(\theta_{t},{ }^{\circ}\right)$ & 60 \\
Inter-recess land-width ratio & 0.33 \\
\hline
\end{tabular}

\subsection{Load capacity analysis}

To describe the attitude of the spindle rotor under external load, the ratio of the displacement of the center point of the front end of the spindle to the oil film thickness is defined as the dimensionless relative eccentricity $\varepsilon_{e}$ :

$$
\varepsilon_{e}=\frac{\sqrt{\left(\Delta x+l_{c} \cdot \Delta \theta_{y}\right)^{2}+\left(\Delta y-l_{c} \cdot \Delta \theta_{x}\right)^{2}}}{C_{0}}
$$

where $\left[\begin{array}{cccc}\Delta x & \Delta y & \Delta \theta_{x} & \Delta \theta_{y}\end{array}\right]$ is the displacement of the center of the spindle rotor at $o-x y z$ coordinate; $l_{c}$ is the distance of the center point of the front end of the spindle to the geometry center of the rotor.

(b)

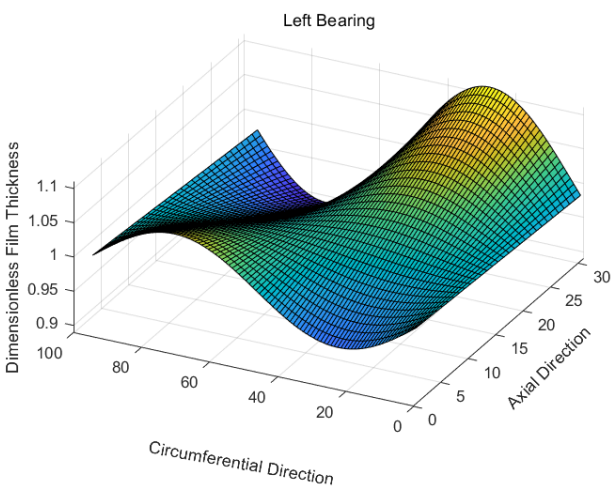

(d)

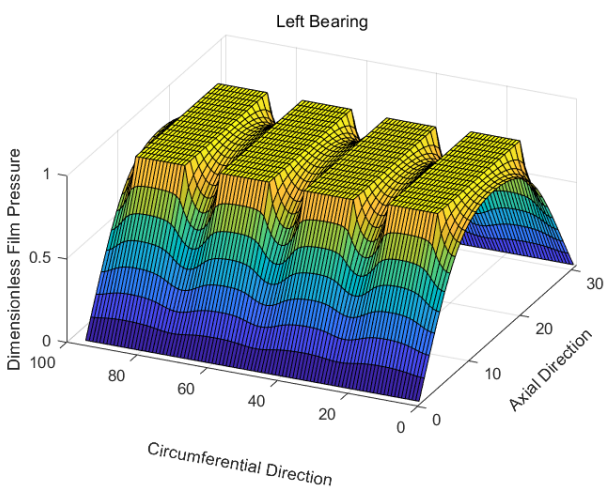


(e)

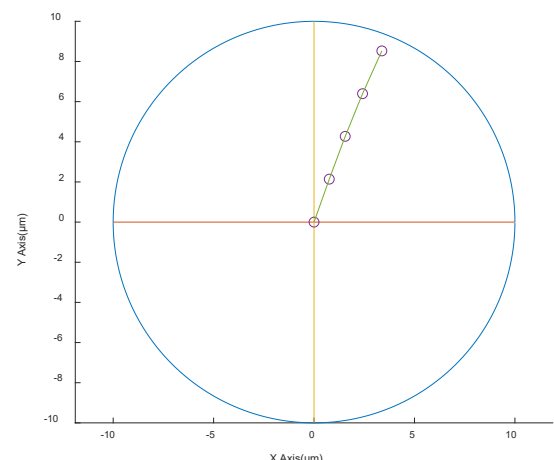

(f)

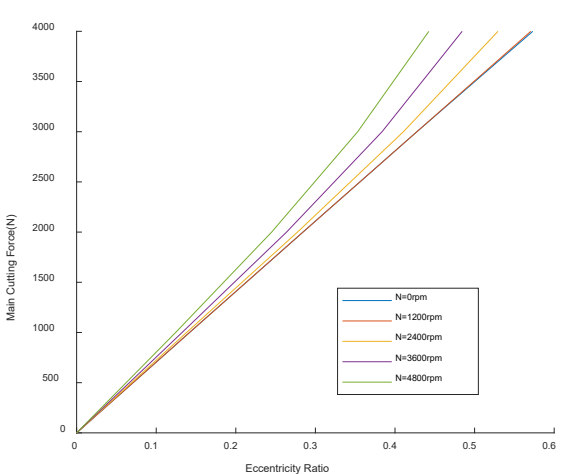

Figure 4. Static characteristics of the hydrostatic spindle $(\omega=0)$. (a) Oil film thickness of right journal bearing; (b) oil film thickness of left journal bearing; (c) pressure distribution of right journal bearing; (d) pressure distribution of left journal bearing; (e) trajectory of rotor; (f) capacity of the spindle.

When the spindle rotor is in a slant positing under external load, the oil film clearance of journal and thrust bearings can be calculated by equations (7) and (8). Figures 4(a) and 4(b) show the distribution of dimensionless oil film thickness of the right and left journal bearings and explain that the oil film thickness is changed sharply due to the rotor's tilt. Since the computation for thrust bearing is somewhat the same as that of journal bearing, no more repeating here.

The pressure distribution, which varies following the change of oil film thickness, can be obtained by solving the Reynolds equation, as shown in Figures 4(c) and 4(d). The dimensionless pressure of each recess is no longer equal because the rotor tilt changes the liquid resistance of the filmland.

Figure 4(e) shows the steady-state equilibrium position of the center point of the front end of the spindle under different

(a)
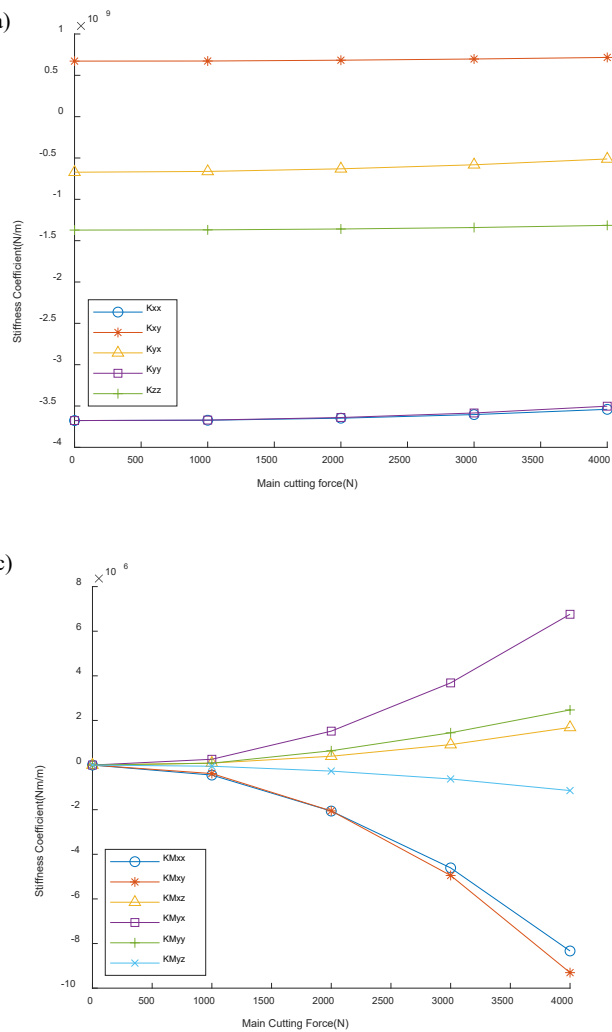

cutting force. When the external load is $0 \mathrm{~N}$, the center of the spindle is at the origin point. With the increase of the cutting load, the spindle rotor center gradually shifted along the radial direction. When the cutting force reached the maximum load of the spindle, which is $4000 \mathrm{~N}$, the spindle center was at $(3.8 \mu \mathrm{m}, 8.7 \mu \mathrm{m})$. Figure $4(\mathrm{f})$ shows the relationship between relative eccentricity ratio and external load under different rotational speeds. When the rotational speed is $0 \mathrm{rpm}$, the load curve is linear, and the eccentricity ratio is 0.58 under the force of $4000 \mathrm{~N}$. Due to the oil film wedging effect, the bearing capacity of the spindle increase with the gradual rise of spindle speed. When the spindle reaches the maximum rotation speed of $4800 \mathrm{rpm}$, the relative eccentricity ratio is 0.45 under the load of $4000 \mathrm{~N}$.

\subsection{Performances versus main cutting force}

(b)

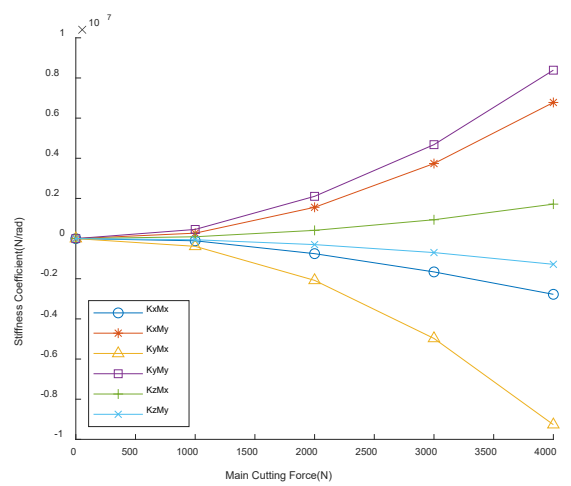

(d)

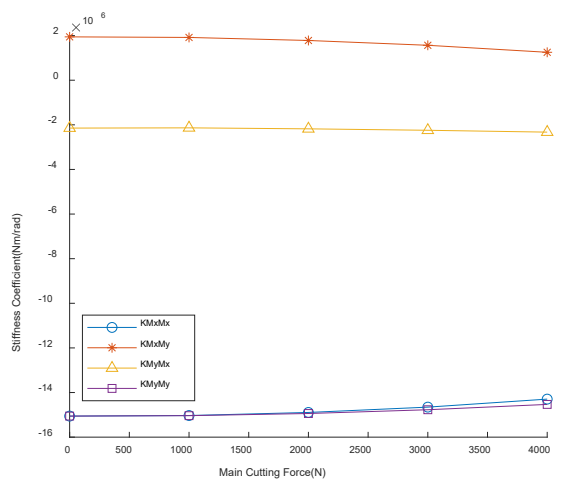




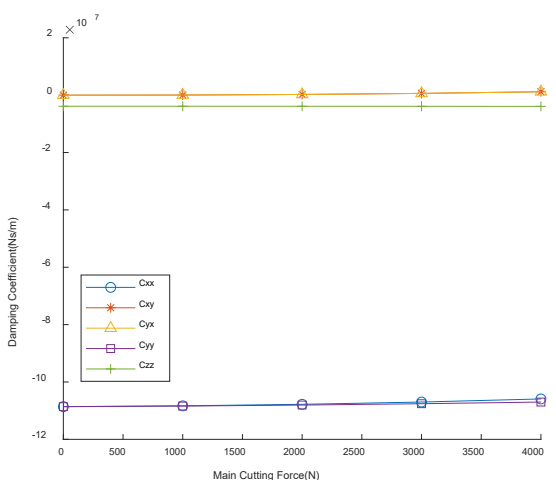

(g)

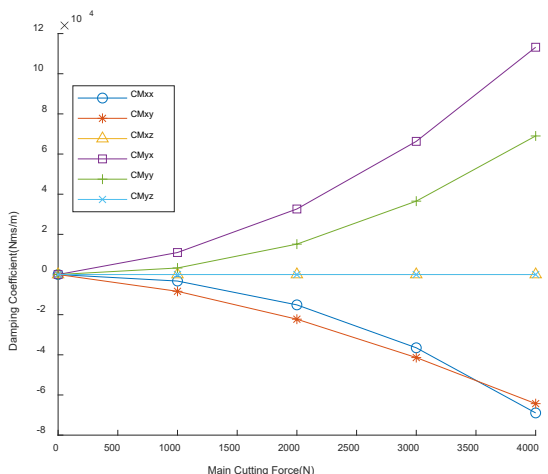

(i)

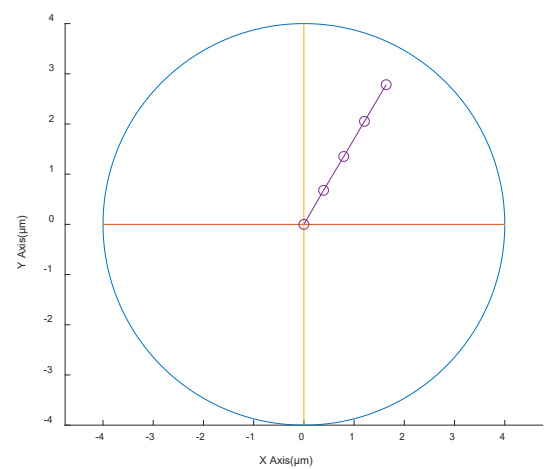

(f)

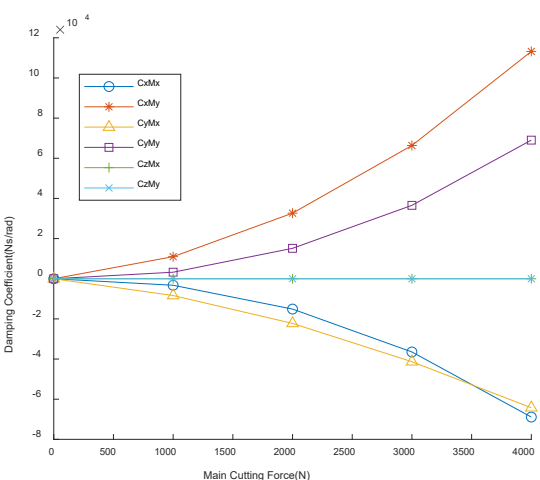

(h)

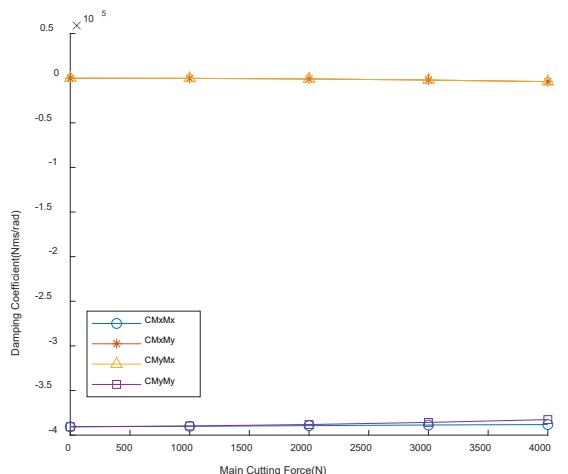

(j)

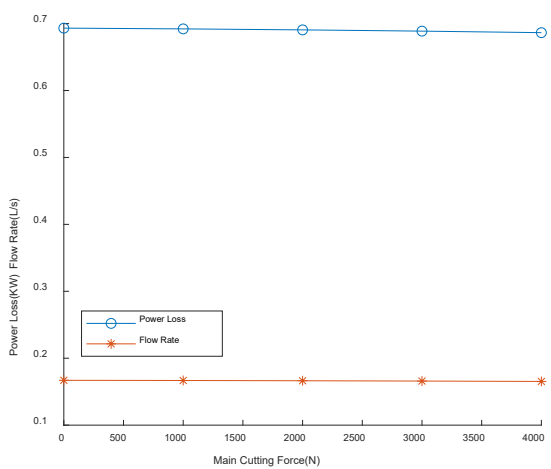

Figure 5. Variation of static and dynamic characteristics with the main cutting force under different amplitudes $(\omega=3000 \cdot 2 \pi)$. (a) Force stiffness coefficients due to translation; (b) force stiffness coefficients due to tilt motion; (c) moment stiffness coefficients due to translation; (d) moment stiffness coefficients due to tilt motion; (e) force damping coefficients due to translation; (f) force damping coefficients due to tilt motion; (g) moment damping coefficients due to translation; (h) moment damping coefficients due to tilt motion; (i) position of center point on the end face of the spindle; (j) power consumption and flowrate.

In Figure 5, the main simulation parameters include the rotating speed of the spindle of which is constant $3000 \mathrm{rpm}$, and the fundamental cutting force of which varies from 0 to 4000 N. Figures 5(a) and 5(d) show that force stiffness coefficients due to translation and moment stiffness coefficients due to tilt motion change little with the increase of main cutting force, especially, the above stiffness has little relation with eccentricity ratio. Also, due to the wedging effect caused by spindle rotation, the cross stiffness $K_{x y}, K_{y x}, K_{\theta_{x} \theta_{y}}$ and $K_{\theta_{y} \theta_{x}}$ are not zero and show a skew-symmetric variation law. Figures 5(b) and 5(c) illustrate that force stiffness coefficients due to tilt motion and moment stiffness coefficients due to translation grow significantly from 0 with the increment of main cutting force; the stiffness on z-axis direction increases slowly. The above phenomena explain that the displacement of the spindle rotor has little influence on the oil film thickness variation of the thrust bearing. The simulation results of damping are similar to that of the stiffness, as shown in Figures 5(e) to 5(h). Force damping coefficients due to translation and moment damping coefficients due to tilt motion variation is negligible with the increase of the main cutting force. In Figures 5(f) and 5(g), force damping coefficients due to tilt motion and moment damping coefficients due to translation increase obviously from 0 with the development of main cutting force except for the damping coefficients on z-axis direction. Besides, the above cross damping coefficients are following a skewsymmetric variation trend.

Figure 5(i) shows that the steady-state position of the spindle rotor changes with the increase of the main cutting force. With the growth of the main cutting force, the displacement of the center point on the end face of the spindle along the $\mathrm{x} / \mathrm{y}$ direction increases linearly. By comparing Figure 5(i) with 
Figure 4(e), it shows that the rotor displacement in Figure 5(i) is much smaller than that in Figure $4(\mathrm{e})$ because of the wedging effect of spindle rotation. Figure 5(j) represents that the power consumption and flowrate change slightly with the

(a)

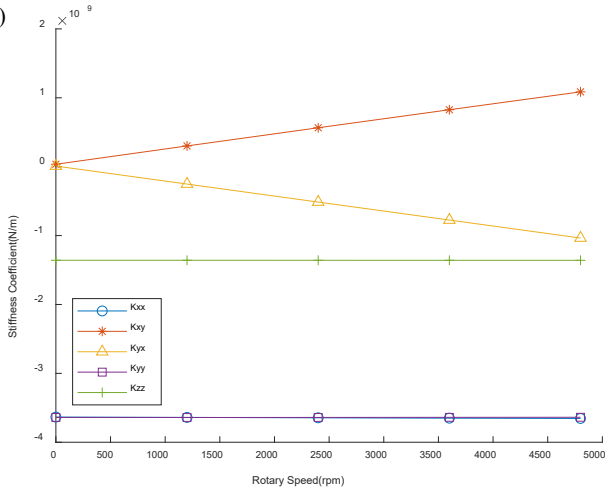

(c)

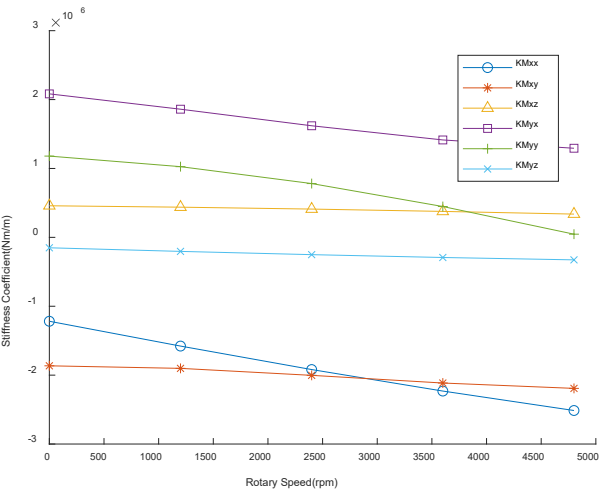

(e)

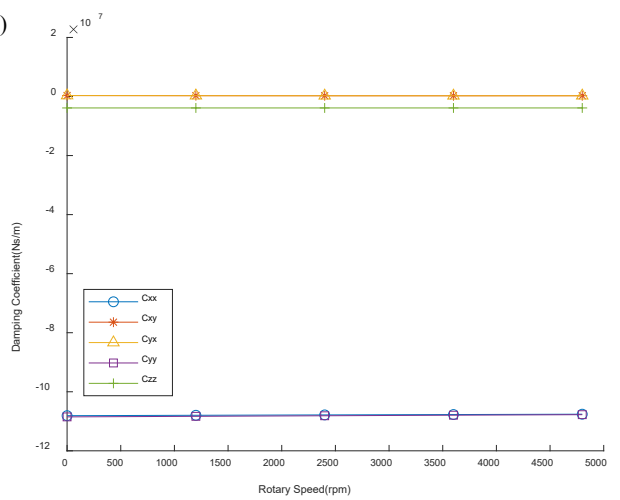

(g)

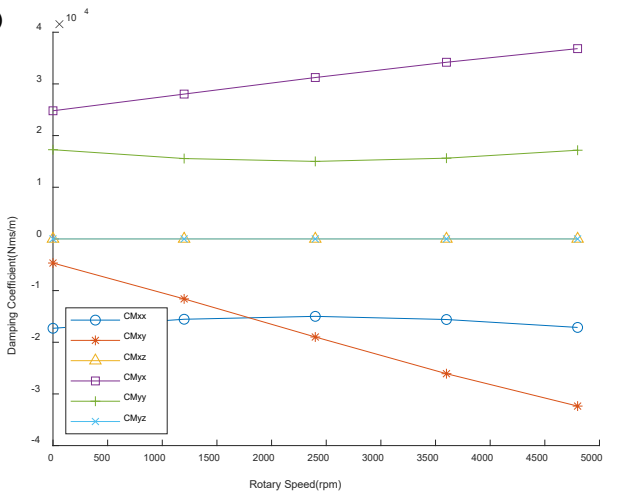

increase of the main cutting force.

3.3 Performances versus rotary speed

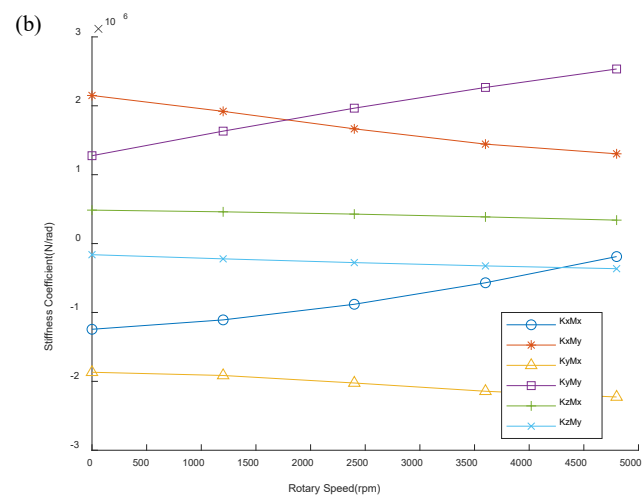

(d)
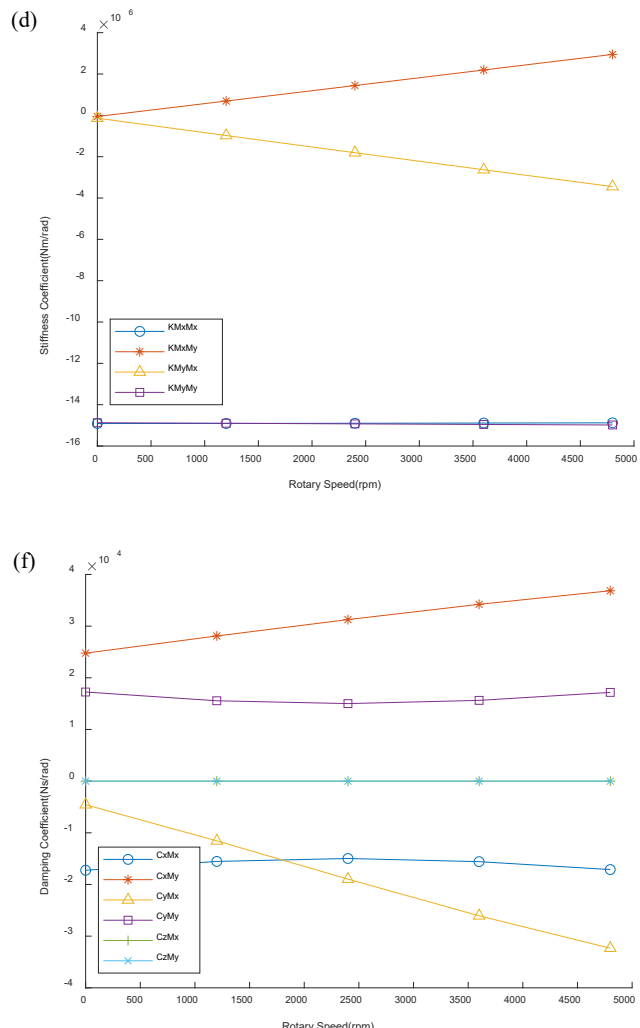

(h)

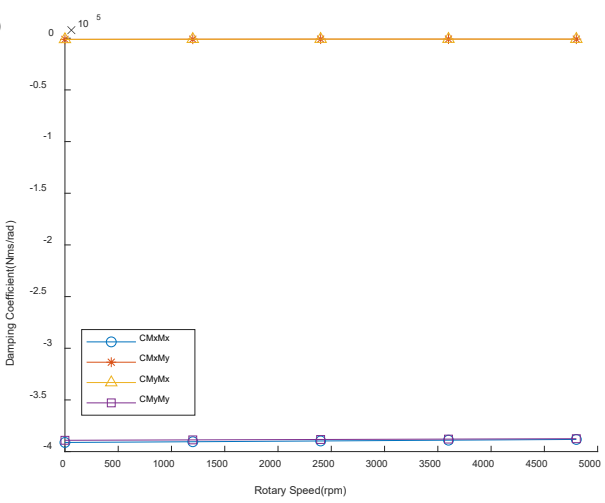


(i)

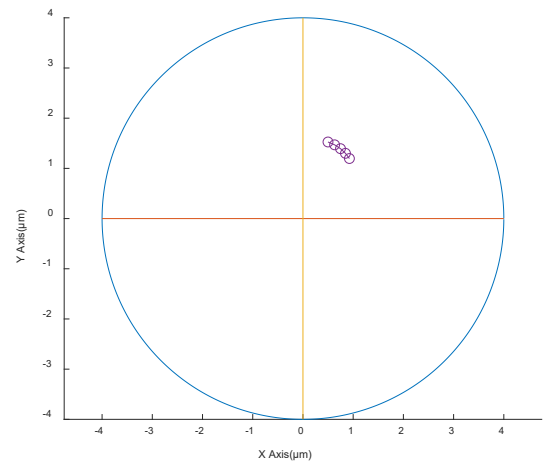

(j)

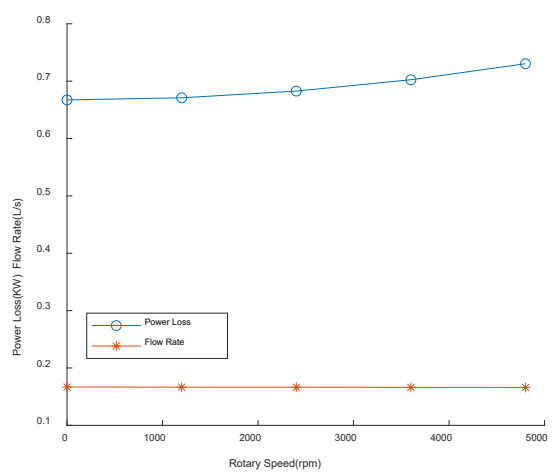

Figure 6. Variation of static and dynamic characteristics with rotary speed $(F=2000 N)$. (a) Force stiffness coefficients due to translation; (b) force stiffness coefficients due to tilt motion; (c) moment stiffness coefficients due to translation; (d) moment stiffness coefficients due to tilt motion; (e) force damping coefficients due to translation; (f) force damping coefficients due to tilt motion; (g) moment damping coefficients due to translation; (h) moment damping coefficients due to tilt motion; (i) position of center point on the end face of the spindle; (j) power consumption and flowrate.

The main simulation parameters include: the main cutting force is constant $2000 \mathrm{~N}$; the spindle speed varies from 0 to $3000 \mathrm{rpm}$. Figures 6(a) and 6(d) illustrate that the principal stiffness coefficients $K_{x x}, K_{y y}, K_{z z}, K_{\theta_{x} \theta_{x}}$ and $K_{\theta_{y} \theta_{y}}$ do not change with the increase of rotary speed. Further, the cross stiffness coefficients $K_{x y}, K_{y x}, K_{\theta_{x} \theta_{y}}$ and $K_{\theta_{y} \theta_{x}}$ increase rapidly following skew-symmetric trend under the same condition due to the oil film wedging effect caused by the increase of rotary speed. In Figures 6(b) and 6(c), the cross stiffness coefficients $K_{x \theta_{x}}, K_{x \theta_{y}}, K_{\theta_{y} x}$ and $K_{\theta_{y} y}$ decrease with the rise of rotary speed, but the variation of the cross stiffness coefficients $K_{y \theta_{x}}, K_{y \theta_{y}}, K_{\theta_{x} x}$, and $K_{\theta_{x} y}$ are in the opposite trend. Also, force stiffness coefficients on z-axis direction change a little with the increase of rotary speed because the effective bearing area of thrust bearing is much less than that of the journal bearing shown in Figure 1. Figures 6(e) to 6(h) represent that damping coefficients $C_{x \theta_{y}}, C_{y \theta_{x}}$, $C_{\theta_{y} x}$ and $C_{\theta_{x} y}$ are growing with the increase of rotary speed, which is also following the skew-symmetric rule. The rest damping coefficients are not sensitive to the change of rotary motion velocity.

In Figure 6(i), with the rotary speed increasing, the relative eccentricity ratio of the spindle rotor is almost constant; the steady-state position of the spindle rotor changes a little along the circumferential direction of the center of the spindle.
Figure $6(j)$ demonstrates that the power consumption of the spindle becomes larger by about $8 \%$ with the increase of rotary speed due to the rise of friction inside the oil film is proportional to the rotation speed of the journal, but the flowrate is constant.

\subsection{Performances versus distance of cutting force}

The main simulation parameters in Figure 7 include: 1, the rotating speed of the spindle is $3000 \mathrm{rpm} ; 2$, the main cutting force is $2000 \mathrm{~N} ; 3$, the cutting distance varies from 0 to $120 \mathrm{~mm}$. Figures 7 (a) and 7 (d) demonstrate that force stiffness coefficients due to translation and moment stiffness coefficients due to tilt motion change slightly with the increase of the cutting distance. They also indicate that the eccentricity ratio of the spindle rotor caused by cutting force has little influence on the above stiffness coefficients. Figures 7 (b) and 7 (c) depict that force stiffness coefficients due to tilt motion and moment stiffness coefficients due to translation grow linearly following the skew-symmetric trend with the increase of the cutting distance except for stiffness coefficients on zaxis direction. The variation trend of damping coefficients is similar to that of stiffness coefficients shown in Figures 7(e) to $7(\mathrm{~h})$, which proves that the cutting distance has a similar effect on the stiffness and damping characteristics of the spindle in a linear way. Due to the tilt of the spindle rotor under cutting force, figure $7(\mathrm{f})$ to $7(\mathrm{~g})$ demonstrate that all cross damping coefficients grow linearly with the increase of the cutting distance except damping coefficients on $\mathrm{z}$-axis direction. Also, $C_{x \theta_{y}}, C_{y \theta_{y}}, C_{\theta_{y} x}, C_{\theta_{y} y}$ are proportional to the cutting distance, but $C_{x \theta_{x}}, C_{y \theta_{x}}, C_{\theta_{x} x}, C_{\theta_{x} y}$ are inversely proportional to the cutting distance.
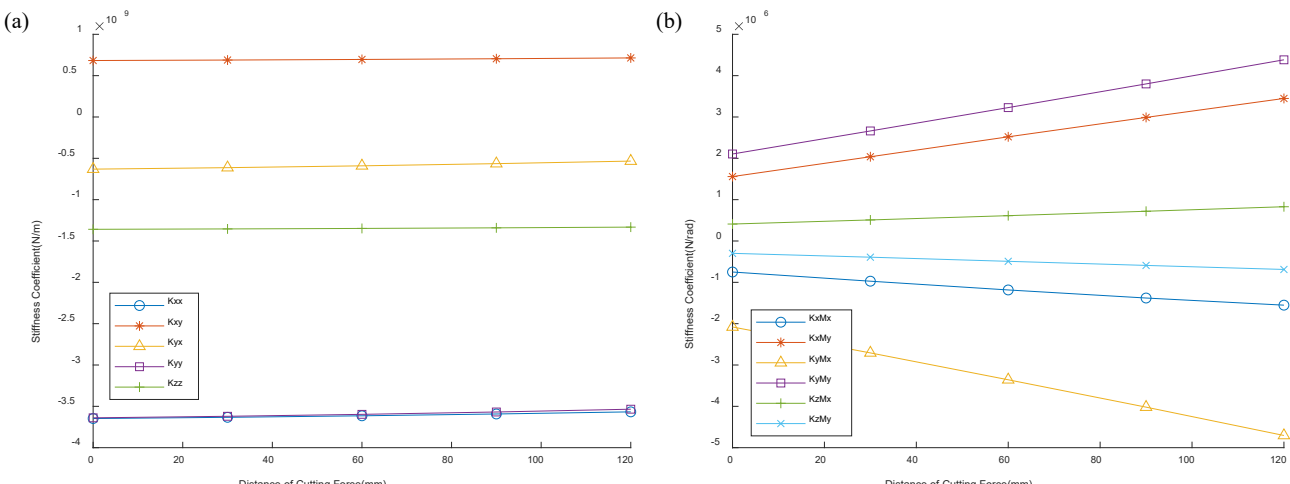
(c)

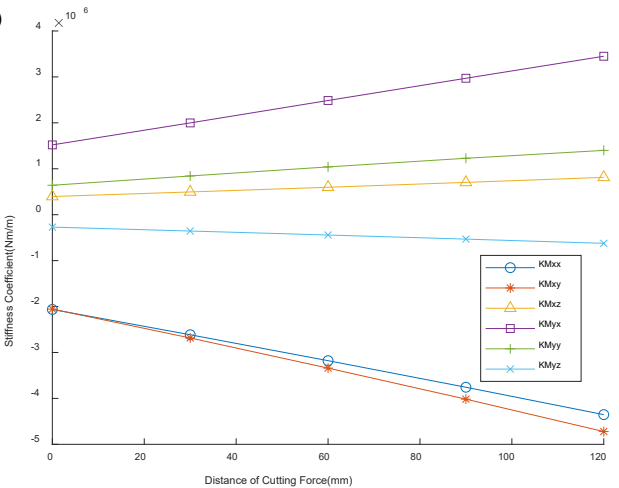

(e)

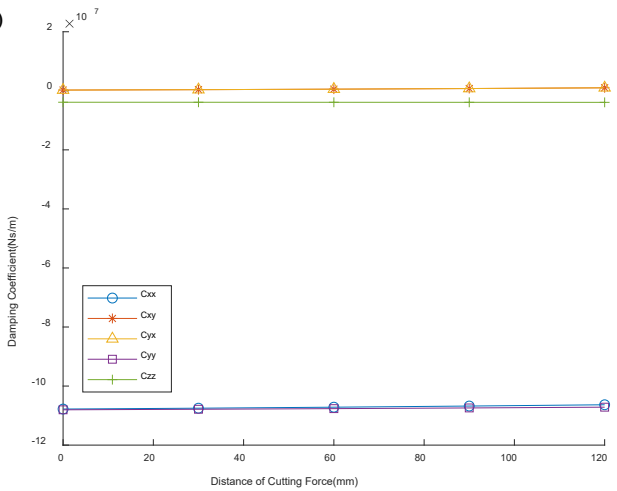

$(\mathrm{g})$
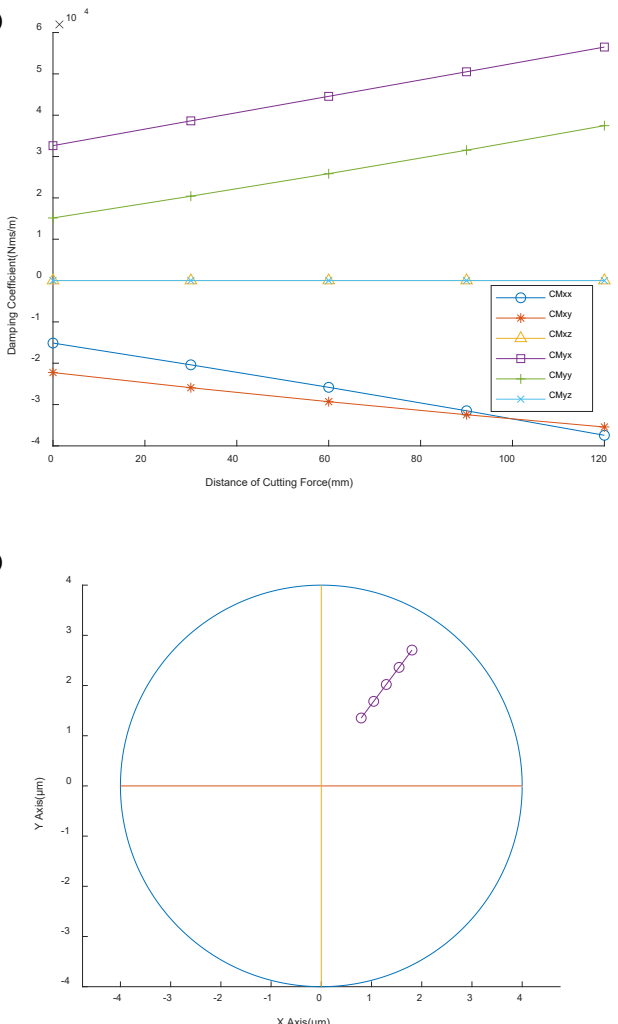

(d)

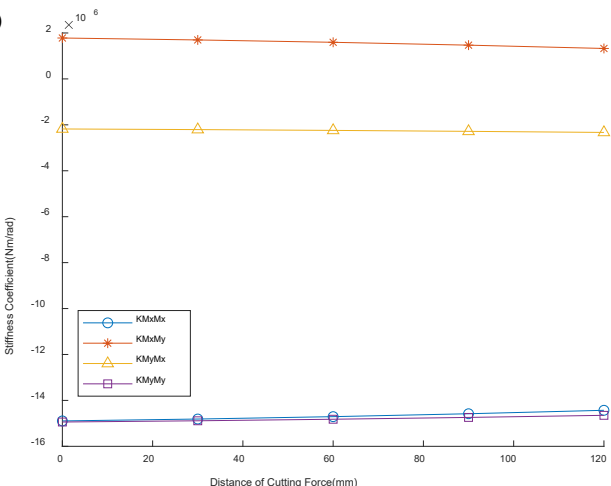

(f)

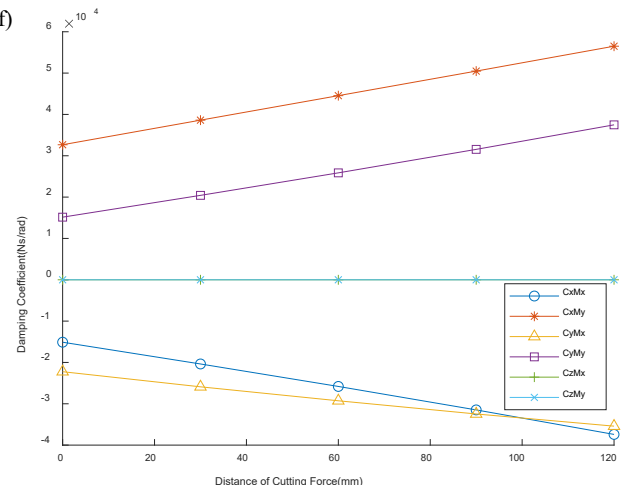

(h)

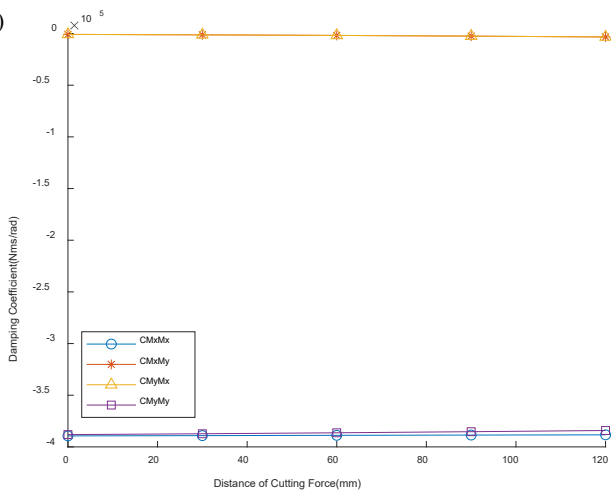

(j)

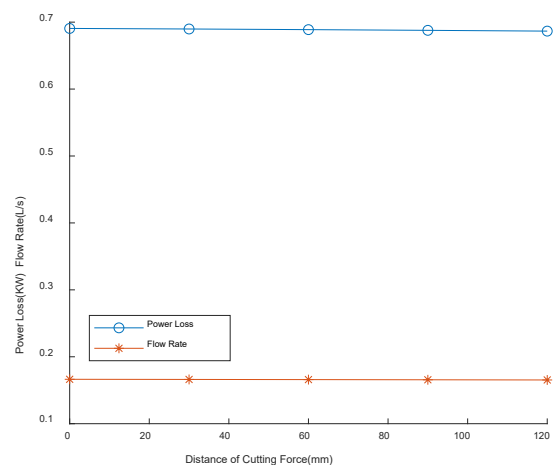

Figure 7. Variation of static and dynamic characteristics with the distance of cutting force ( $\mathrm{F}=2000 \mathrm{~N}, \omega=3000 \cdot 2 \pi)$. (a) Force stiffness coefficients due to translation; (b) force stiffness coefficients due to tilt motion; (c) moment stiffness coefficients due to translation; (d) moment stiffness coefficients due to tilt motion; (e) force damping coefficients due to translation; (f) force damping coefficients due to tilt motion; (g) moment damping coefficients due to translation; (h) moment damping coefficients due to tilt motion; (i) position of center point on the end face of the spindle; (j) power consumption and flowrate. 
Similar to the influence of the main cutting force, with the increase of the cutting distance, the steady-state position of the center of the spindle end face moves outward along the radial direction, whose eccentricity is proportional to the cutting distance, as shown in Figure $7(\mathrm{i})$. Figure $7(\mathrm{j})$ represents the power consumption and flowrate almost keep constant with the increase of the cutting distance, which indicates that the power consumption and flowrate are insensitive to the tilt of the spindle rotor.

\subsection{Stability analysis}

Figure 8 mainly reflects the relationship between the stability of the spindle and the cutting force and its operating distance. The curves in Figure 8(a) are the relationships among the cutting force, the cutting distance, and the whirl ratio. The stable region is below the curve, and the unstable region is above the curve. It also shows that the whirl ratio decreases

(a)

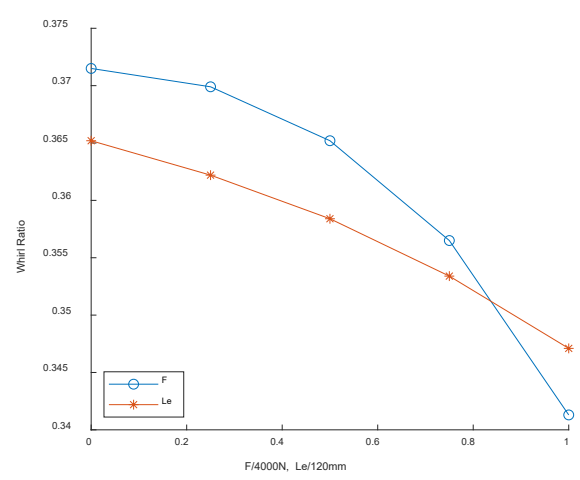

gradually with the increase of cutting force and the cutting distance, but the variation range is small. The curves in Figure 8(b) represent the relationship among the cutting force, the cutting distance, and the dimensionless critical speed. This figure shows that with the increase of the cutting force and the cutting distance, the main critical speed increases gradually. Besides, Figure 8 shows that the stability threshold decided by the change of cutting force is higher than that of the cutting distance. Furthermore, based on the analysis of Figures (5) and (7), it is found that the comprehensive stiffness coefficients and damping coefficients of the spindle increase with the rise of cutting force and the cutting distance simultaneously. Therefore, the stability threshold of the spindle varies little within the whole working range, and its dimensionless critical speed $\bar{\omega}$ is far less than the allowable maximum working speed of the spindle.

(b)

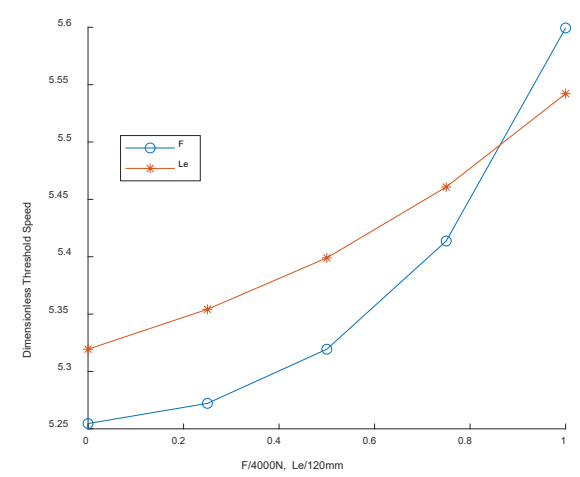

Figure 8. Stability analysis of spindle. (a) Whirl ratio; (b) dimensionless threshold speed.

\section{Conclusions}

This paper establishes a precise hydrostatic spindle model with mid-thrust bearings, analyzes the bearing capacity, stiffness, damping, and stability of the spindle, and studies the dynamic and static performance characteristics of the spindle under different working loads. From the investigation, several conclusions can be drawn as follows:

(1) The spindle rotor generates a five degree-of-freedom displacement under the effect of working load, and its end bearing capacity is directly proportional to the relative eccentricity rate and the spindle rotation speed.

(2) With the increase of the cutting load, the relative eccentricity of the spindle increases linearly; the variation amplitude of principal stiffness and damping is small, and the increased amplitude of cross stiffness and damping is evident. (3) With the increase of spindle speed, the relative eccentricity ratio of the spindle remains unchanged, and the principal stiffness and damping almost keep constant, but the amplitude of cross stiffness and damping changes significantly. Furthermore, the power consumption of the spindle increases by about $15 \%$ within the operating range of speed.

(4) As the operating distance of the load on the spindle rotor increases, the relative eccentricity of the spindle increases linearly; the principal stiffness and damping remain constant; the cross stiffness and damping increment linearly.

(5) The dimensionless critical speed $\bar{\omega}$ of the spindle rotor increases with the increase of external load and cutting distance. But within the operating range of the spindle, the critical steady velocity is far higher than the allowable maximum operating speed of the spindle.

Declaration of Conflicting Interests

The author(s) declared no potential conflicts of interest with respect to the research, authorship and/or publication of this article.

\section{Funding}

This work was supported by the National Natural Science Foundation of China (Grant Nos. 51635003).

\section{Appendix:}

\section{Nomenclature}

$A_{r}$ recess area;

$C_{0}$ design oil film thickness of the journal and thrust bearing; $C, \bar{C}$ damping matrix of spindle and its dimensionless form; $\bar{C}_{j r}, \bar{C}_{j l}, \bar{C}_{t r}, \bar{C}_{t l}$ dimensionless damping matrix of each journal and thrust bearing;

$\bar{c}_{i j}$ : dimensionless damping coefficients $\left(i, j=x, y, z, \theta_{x}, \theta_{y}\right)$;

$d_{c}$ diameter of orifice restrictor;

D diameter of journal bearing, $R=D / 2$;

$F_{x}, F_{y}, F_{z}, M_{x}, M_{y}, \bar{F}_{x}, \bar{F}_{y}, \bar{F}_{z}, \bar{M}_{x}, \bar{M}_{y} \quad$ oil film force and moment with their dimensionless form;

$F_{x e}, F_{y e}, F_{z e}$ cutting force and moment;

$h, \bar{h} \quad$ oil film thickness and its dimensionless form;

$H_{f} \quad$ friction power $\left(H_{f}=\frac{\eta\left(\pi D L-0.75 A_{r}\right)}{C_{0}}\right)$;

$H_{p} \quad$ pumping power $\left(H_{p}=p_{s} q\right)$;

$I_{x}, I_{y}, I_{z}$ the mass moments of inertia of the spindle's rotor; $K, \bar{K} \quad$ stiffness matrix of spindle and its dimensionless form; $K_{p} \quad$ concentric power ratio $\left(K_{p}=\frac{H_{f}}{H_{p}}\right)$;

$\bar{K}_{j r}, \bar{K}_{j l}, \bar{K}_{t r}, \bar{K}_{t l} \quad$ dimensionless stiffness matrix of each journal and thrust bearing; 
$\bar{k}_{i j} \quad$ dimensionless stiffness coefficients $\left(i, j=x, y, z, \theta_{x}, \theta_{y}\right)$;

$K_{e q}, \bar{K}_{e q}$ equivalent stiffness and its dimensionless form;

$l_{e}$ the distance of cutting force to the center of rotor:

$l_{c}$ the distance of the end face of spindle to the center of rotor;

$L$ Bearing axial length;

$\mathrm{m}$ the mass of rotor;

$p, \bar{p} \quad$ oil film pressure and its dimensionless form;

$p_{r}$ absolute recess pressure;

$p_{s} \quad$ oil supply pressure;

$Q_{i n}, \bar{Q}_{i n}$ inflow rate of recess and its dimensionless form;

$Q_{\text {out }}, \bar{Q}_{\text {out }}$ outflow rate of recess and its dimensionless form;

$r_{i}, \bar{r}_{i} \quad$ radius of thrust bearing and its dimensionless form;

$R_{1}, R_{4} \quad$ inner and outer radius of thrust bearing

$S_{h}$ velocity coefficients of journal bearing;

$S_{t} \quad$ velocity coefficients of thrust bearing;

$t, \bar{t}$ times, $\bar{t}=\omega t$

$U$ velocity on the surface of journal bearing;

$x, y, z, \theta_{x}, \theta_{y} \quad$ Cartesian coordinates;

$\bar{Z}$ dimensionless coordinate of axial distance;

$z_{i}, \bar{Z}_{i} \quad$ the axial distance from the rotation center and its dimensionless form;

$\alpha$ flow coefficient of orifice restrictor;

$\epsilon$ relaxation factor;

$\beta$ concentric pressure ratio $\left(\beta=\frac{p_{r}}{p_{s}}\right)$;

$\gamma$ whirl ratio;

$\lambda$ eigenvalue of the motion equation of spindle's rotor;

$\sigma$ the real part of $\lambda$;

$\delta$ dimensionless orifice restrictor coefficient;

$\omega, \bar{\omega} \quad$ rotary speed of spindle and its dimensionless form;

$\omega_{s}$ journal perturbation rotating speed;

$\varepsilon_{e}$ relative eccentricity ratio

$\rho$ density of oil;

$\eta \quad$ viscosity of oil;

$\theta$ dimensionless circumferential direction;

$\Delta p$ pressure difference of orifice restrictor;

$\Delta x, \Delta y, \Delta z, \theta_{x}, \theta_{y}, \Delta \bar{x}, \Delta \bar{y}, \Delta \bar{z} \quad$ the displacement of spindle's rotor and their dimensionless form;

$\xi=x, y, z, \theta_{x}, \theta_{y} ;$

$\zeta=0, x, y, z, \theta_{x}, \theta_{y}, \dot{x}, \dot{y}, \dot{z}, \dot{\theta}_{x}, \dot{\theta}_{y}$.

\section{Reference}

1. Ryszard Przybyẽl. Some Aspects of Application of the Hydrostatic Bearings in Machine Tools Mechanics and Mechanical Engineering. 2008; Vol. 12:243-253.

2. Feng Cheng, Weixi Ji. Simultaneous identification of bearing dynamic coefficients in a water-gas lubricated hydrostatic spindle with a big thrust disc. Journal of Mechanical Science and Technology. 2016;30 (9):4095 4107.

3. B. Knapp, D. Arneson, D. Oss. Ultra-precision, High Speed Micromachining Spindle. Proceedings of the 11th euspen International Conference. May 2011.

4. Bo-Sung Kim, Gyeong-Tae Bae, Gwi-Nam Kim. A study on the thermal characteristics of the grinding machine applied hydrostatic bearing. Transactions of the Canadian Society for Mechanical Engineering. 2015; 39(3): 717-728.

5. Dmytro Fedorynenko, Rei Kirigaya, Yohichi Nakao. Dynamic characteristics of spindle with water-lubricated hydrostatic bearings for ultra-precision machine tools. Precision Engineering. 2020; 63:187-196.

6. Yohichi NAKAO, Yuji SAGESAKA. Development of Water Drive Spindle. Proceedings of the 6th JFPS International Symposium on Fluid Power, TSUKUBA. November 2005.
7. G.H.Jang, Y.J.Kim. Calculation of Dynamic Coefficients in a Hydrodynamic Bearing Considering Five Degrees of Freedom for a General Rotor-Bearing System. Journal of Tribology. 1999; Vol. 121: 499-505.

8. Lund, J. W., and Thomsen, K. K.. A Calculation Method and Data for the Dynamic Coefficients of Oil-Lubricated Journal Bearing. Topics in Fluid Film Bearing and Rotor Bearing System Design and Optimization, ASME, New York, 1978; 1-28.

9. V. Castelli, W. Shapiro. Improved Method for Numerical Solutions of the General Incompressible Fluid Film Lubrication Problem. J. of Lubrication Tech. Apr 1967;89(2): 211-218.

10. Jialei DU, Guozhu LIANG. Dynamic coefficients and stability analysis of a water-lubricated hydrostatic bearing by solving the uncoupled Reynolds equation. Chinese Journal of Aeronautics. 2020;33(8):2110-2122.

11. Huihui Feng, Shuyun Jiang. Dynamic analysis of waterlubricated motorized spindle considering tilting effect of thrust bearing. J Mechanical Engineering Science. 2017; Vol. 231(20): 37803790.

12. Jiasheng Li, Pinkuan Liu. Dynamic analysis of 5-DOFs aerostatic spindles considering tilting motion with varying stiffness and damping of thrust bearings. Journal of Mechanical Science and Technology. 2019;33 (11) : 5199 5207.

13. Jianghai Shi, Hongrui Cao, Xuefeng Chen. Effect of angular misalignment on the dynamic characteristics of externally pressurized air journal bearing. September 2019 Vol.62 No.9: 1520 1533.

14. Abdurrahim Dal, Tuncay Karaçay. Effects of angular misalignment on the performance of rotor-bearing systems supported by externally pressurized air bearing. Tribology International. 2017;111:276-288.

15. Myunggyu Kim, Gunhee Jang, HakwoonKim. Stability analysis of a disk-spindle system supported by coupled journal and thrust bearings considering five degrees of freedom. Tribology International. 2010;43:1479-1490.

16. Hakwoon Kim, Gunhee Jang, Sanghoon Lee. Complete determination of the dynamic coefficients of coupled journal and thrust bearings considering five degrees of freedom for a general rotor-bearing system. Microsyst Technol. 2011;17:749-759.

17. Gunhee Jang* and Sanghoon Lee. Determination of the dynamic coefficients of the coupled journal and thrust bearings by the perturbation method. Tribology Letters. June 2006;Vol. 22, No. 3: 239-246.

18. Hakwoon Kim, GunheeJang n, HeonjeongHa. A generalized Reynolds equation and its perturbation equations for fluid dynamic bearings with curved surfaces. Tribology International. 2010:43:1479-1490.

19. Panagiotis Koutsovasilis. Automotive turbocharger rotor dynamics: Interaction of thrust and radial bearings in shaft motion simulation. Journal of Sound and Vibration0 2019;455:413-429.

20. Shuyun Jiang, Shengye Lin, Chundong Xu. Static and Dynamic Characteristics of Externally Pressurized Porous Gas Journal Bearing With Four Degrees-of-Freedom. Journal of Tribology. JANUARY 2018; Vol. 140 / 011702:1-13.

21. Yongtao Zhang, , Shijie Yu, Changhou Lu. An improved lumped parameter method for calculating static characteristics of multirecess hydrostatic journal bearings. Proc IMechE Part J:J Engineering Tribology. 2020; Vol. 234(2): 301-310.

22. Wenjie Zhou, XuesongWei, LeqinWang. A superlinear iteration method for calculation of finite length journal bearing's static equilibrium position. R. Soc. open sci.4: 161059.. May 2017;1-19.

23. Yoshimi Ito, Takashi Matsumura. Theory and Practice in Machining Systems. Springer International Publishing Switzerland, 2017.

24. Laís Bittencourt Visnadi, Helio Fiori de Castro. Influence of bearing clearance and oil temperature uncertainties on the stability threshold of cylindrical journal bearings. Mechanism and Machine Theory. 2019;134:57-73.

25. Tianming Ren, Ming Feng. Stability analysis of water-lubricated journal bearings for fuel cell vehicle air compressor. Tribology International. 2016;95:342-348.

26. Rowe, F S Chong. Computation of Dynamic Force Coefficients for Hybrid Hydrostatic/Hydrodynamic Journal Bearings by the FD technique and by the Perturbation technique. Tribology International. Oct 1986; 19(5); 260-271. 